\title{
An Innovative Approach to Clinical Practice Guideline Adaptation in the Nursing Profession in a Developing Country
}

This article was published in the following Dove Press journal: Cancer Management and Research

\author{
Shahin Salarvand, (D) \\ Simin Hemati, (iD ${ }^{2}$ \\ Payman Adibi, ${ }^{3}$ \\ Fariba Taleghani, ${ }^{4}$ \\ Mohammad Saleki ${ }^{5}$ \\ 'Hepatitis Research Center, \\ Community Health Nursing \\ Department, Faculty of Nursing and \\ Midwifery, Lorestan University of \\ Medical Sciences, Khorramabad, Iran; \\ ${ }^{2}$ Radiotherapy and Oncology \\ Department, Faculty of Medicine, \\ Isfahan University of Medical Sciences, \\ Isfahan, Iran; ${ }^{3}$ Gastroenterology and \\ Hepatology Department of Internal \\ Medicine, School of Medicine, \\ Integrative Functional \\ Gastroenterology Research Al-Zahra \\ Hospital, Isfahan University of Medical \\ Sciences, Isfahan, Iran; ${ }^{4}$ Nursing and \\ Midwifery Care Research Center, \\ Faculty of Nursing and Midwifery, \\ Isfahan University of Medical Sciences, \\ Isfahan, Iran; ${ }^{5}$ Department of Sport \\ Medicine, Isfahan University of Medical \\ Sciences, Isfahan, Iran
}

Correspondence: Shahin Salarvand Hepatitis Research Center, Community Health Nursing Department, Faculty of Nursing and Midwifery, Lorestan University of Medical

Sciences, Khorramabad, Iran

Tel +989161590560

Fax +986633120140

Email shsalarvand@lums.ac.ir
Introduction: Healthcare professionals should seek the necessary resources to improve the quality of care. Given the cultural, social, and economic differences, in every health care system, there are increasing needs for the adapted versions of clinical practice guidelines (CPGs). This study aimed to introduce an innovative CPG adaptation approach for nurses working in a developing country.

Methods: This study is comprised of three sections: a. An extensive systematic search of the literature, $b$. The adaptation process, $c$. Interviews, which were held with stakeholders, users and/or the target population. We applied all of the stages of guideline adaptation process according to Adaptation Resource Toolkit, with the integration of the findings of a systematic literature search and a qualitative content analysis in an "adolopted" new CPG.

Results: In this study, we applied a mix of three methods namely adoption, adaptation and development (adolopment) of recommendations to save time, cost, and manpower efficiently. Moreover, we integrated the utilized qualitative research method and literature review with the adolopment approach to develop the recommendations.

Conclusion: Given there is a paucity of nursing clinical practice guidelines (NCPGs) in the nursing management of cancer therapy-induced mucositis and to save time and costs, the findings emerging from the adoption, adaptation, and de novo guideline development by a panel of experts and qualitative content analysis (QCA) method were integrated to achieve a more comprehensive nursing practice guideline.

Keywords: guideline adaptation, developing country, nursing

\section{Introduction}

The nursing profession plays a vital role in implementing educational interventions based on the unique needs of patients and their families, which can help them cope with treatment complications and the disease. In addition, nurses offer professional support in every stage of survival by giving priority to self-care education and focusing on adapting with the activities of daily living and social life. ${ }^{1}$ Hence, nurses should seek the necessary resources to improve the quality of care. This is possible by integrating evidence-based protocols into health care systems. ${ }^{2}$ Nursing managers and administrators should emphasize the provision of evidence-based care as a priority for nurses and they should support nurses and other staff who are involved in cancer care in their efforts. ${ }^{3}$

Nursing clinical practice guidelines (NCPGs) are statements systematically developed based on the best available evidence to assist nurses and patients in deciding to carry out the most appropriate and effective nursing care in special clinical situations 
and can cover all aspects of care from prevention to self-care and interventions. ${ }^{4}$ Additionally, these guidelines present the procedural steps of clinical practice and can help prevent non-standardized clinical practice. ${ }^{5}$

Clinical practice guidelines (CPGs), as a summary of the best scientific evidence, are increasingly being applied in the health care professions to provide evidence-based recommendations for guiding patients' clinical care, which are useful in guiding clinical decision-making. ${ }^{6}$ CPGs are a set of recommendations for multi-disciplinary care teams and are important tools for disseminating the best evidence-based practices. ${ }^{7}$ Developed based on expert opinion and the systematic assessment of evidence about different treatment procedures, CPGs are tools available to health care providers and clients to improve the quality of care. ${ }^{8}$ To promote the health care quality, the ultimate objective of CPGs is to improve the efficiency and costeffectiveness of the health care system and to reduce costly and preventable errors all over the world. ${ }^{9}$

Since the development of CPGs requires financial resources and experts' skills, ${ }^{10,11}$ CPGs can be adapted according to native conditions and local environments to standardize actions and optimize resource utilization. ${ }^{12}$ The trans-contextual adaptation of CPGs is a systematic process for the application and/or modification of CPGs developed in a specific cultural and organizational setting for transfer to a different context. The main objective of the adaptation process is to use the advantages of the extant CPGs for the effective production and application of high-quality adapted CPGs, because a CPG developed for a specific situation may not be appropriately applicable to another context without paying attention to the dimensions and characteristics of the new target setting. ${ }^{13}$

Guideline adaptation is an approach to designing guidelines in a local context with the consensus of the relevant decision-makers. In other words, the existing CPGs can be appraised and modified suitably in accordance with the local context through a dynamic, systematic and cooperative process. This process should maintain the integrity of evidencebased recommendations in that the organizational, local and cultural differences may create considerable variations in guideline recommendations. ${ }^{12}$

In every health care system, there are increasing needs for adapted versions of CPGs because of cultural, social, and economic differences. Given the large volume of content and the high speed of change in medical knowledge, in some cases it may not be possible to develop guidelines de novo, but international guidelines can be used for adaptation $;{ }^{14}$ therefore, the trans-contextual adaptation of CPGs is increasingly applied as an alternative to developing new $\mathrm{CPGs}^{15}$ as we can adapt CPGs according to local settings for cost-effectiveness and because experts from various fields may not be present in the locality. ${ }^{12}$

Hundreds of groups, such as departments, hospitals, other health care agencies, and insurance organizations, specialty faculties, and even small groups, have tried to develop CPGs for improving local clinical practice. They often use their interpretations of the best methods to compile and write CPGs. From the literature review, it can be understood that there are no available detailed processes and standard procedures for guideline development. ${ }^{16}$

The involvement of clinicians and patient stakeholders in the development of evidence-based CPGs requires considerable efforts. ${ }^{17}$ The oncology CPGs recommend every patient, who has recently been diagnosed with cancer, should be managed by a special clinical nurse from the time of the diagnosis of the disease. ${ }^{4,18}$ On the other hand, the importance of evidence-based care using CPGs and the paucity of NCPGs in this area gives rise to the necessity of CPG adaptation for the nursing profession in our country.

At present, the development and implementation of nonpharmacological nursing management CPGs is a priority all over the world. Although there is increasing consensus about methods for developing evidence-based guidelines, this study introduces a method of nursing guideline adaptation that was applied in a developing country. ${ }^{19} \mathrm{We}$ intend to present the applied methodology in this study and avoid to provide any recommendations. This study aimed to introduce an innovative clinical practice nursing guideline adaptation approach in a developing country.

\section{Researcher's Paradigm}

The main researcher (SS) had a pragmatic paradigm due to the concern to collect all the desired content and information for the compilation of clinical guidelines with richer recommendations. Having a pragmatic perspective, the main researcher believes the research question is so important that it cannot be addressed by one specific method. The researcher's pragmatic view helped her in conducting this study as the adaptation process was comprised of several steps and given that she was trying to create a more comprehensive CPG development model, she also applied other methods such as qualitative content analysis (QCA) and a systematic search of the literature for completing recommendations regarding CPG design. In other words, the researcher tried to study the considered phenomenon from different aspects and provide broader, 
deeper and clearer recommendations for CPG design, as the deficient areas of the recommendations were completed by seeking the preferences, views, and perspectives of the patients, health care professionals, and patients' families.

\section{Methods}

This study is comprised of three sections: a. an extensive systematic search of the literature, $b$. the adaptation process, c. interviews, which were held with stakeholders, users and/or the target population. We applied all of the stages of guideline adaptation process according to Adaptation Resource Toolkit, with the integration of the findings of a systematic literature search and a qualitative content analysis in an "adolopted" new CPG.

Guideline developers can select one of these methods: 1 . To adopt extant recommendations without modifications, 2. To adapt extant recommendations according to their own context of use, 3 . To develop new recommendations. ${ }^{20}$

In this study, to save time, cost, and manpower efficiently, we applied a mix of three methods, namely the adoption, adaptation, and development of the recommendations. Moreover, for the first time, the findings of a qualitative study were integrated with a mix of the three aforementioned methods for recommendation design in the preset study. Eventually, an adapted CPG was developed for nurses working in the country. It is worth noting that there had not been any special CPGs for the nursing management of cancer therapy-induced mucositis. Therefore, medical CPGs involving non-pharmacological interventions were applied. Then, these interventions were extracted from the extant medical CPGs and the gaps, where the needed recommendations were not found in current guidelines, were filled with the findings of the qualitative phase of the study, literature review, a survey conducted on experts' views, and finally a panel discussion within the adaptation process (Figure 1). It was attempted to produce a perfect guideline, we collected every related non-pharmacological recommendation or evidence. But we believe there is no a perfect guideline in anywhere.

The main framework of this study is based on the process recommended by The ADAPTE Collaboration Network titled "Guideline Adaptation: A Resource Toolkit". 13 The ADAPTE Collaboration Network is an international collaborative network of researchers and guideline developers, the aim of which is to promote the development and application of CPGs through the adaptation of existing CPGs. The main objective of this group is to produce and validate the adaptation process, which assists in the development of high-quality local guides. Additionally, this group tries to enhance the sense of ownership of consumers over the adapted guideline by involving all the stakeholders in the process. ${ }^{13}$

The adaptation process contains three stages, each stages consisting of several modules, and each module being made up of several steps as follows:

1. Set-up phase, 2. Adaptation phase and 3. Finalization phase.

\section{Set-Up Phase}

This stage consists of the preparation module and the measures conducted before starting the adaptation process, which includes six steps:

\section{The Assessment of the Feasibility of Adaptation}

Before the preparation of the proposal, the existing related CPGs were searched and it was determined that this adaptation process was feasible in our country. Although there were no nonpharmacological and nursing recommendations in other countries' CPGs that could be used in the adaptation process, in order to find published guidelines from the observation time until Dec. 2018, a comprehensive systematic search was conducted on related websites and databases, including SIGN (Scottish Intercollegiate Guidelines Network), NGC (National Guideline Clearinghouse), NCCN (National Comprehensive Cancer Network), NICE (National Institute for Clinical Excellence), CINAHL (Cumulative Index to Nursing and Allied Health Literature), CCO (Cancer Care Ontario), ONS (Oncology Nursing Society), G-IN (Guidelines International Network), NHSC (National Health Service Corps), Cochrane Library, Science Direct, Scopus, PubMed, ProQuest, Google Scholar, Yahoo, Google, ESMO (European Society for Medical Oncology), AHRQ (Agency Health Care Research and Quality), Canadian Partnership against Cancer, WGO (World Gastroenterology Organization), CCMB (Cancer Care Manitoba), and BMJ Best Practice.

\section{Search Strategy to Find Relevant Documents}

The search strategy was conducted by applying keywords in the English language Table 1.

As mentioned, since the design and implementation of CPGs is a priority for health policymakers in Iran, related organizations offer their support and sponsorship to facilitate the adaptation process.

\section{The Determination of a Coordinating Committee}

The organizing committee should supervise the adaptation process. This committee is responsible for some tasks, including specifying the project scope, forming workgroups, 


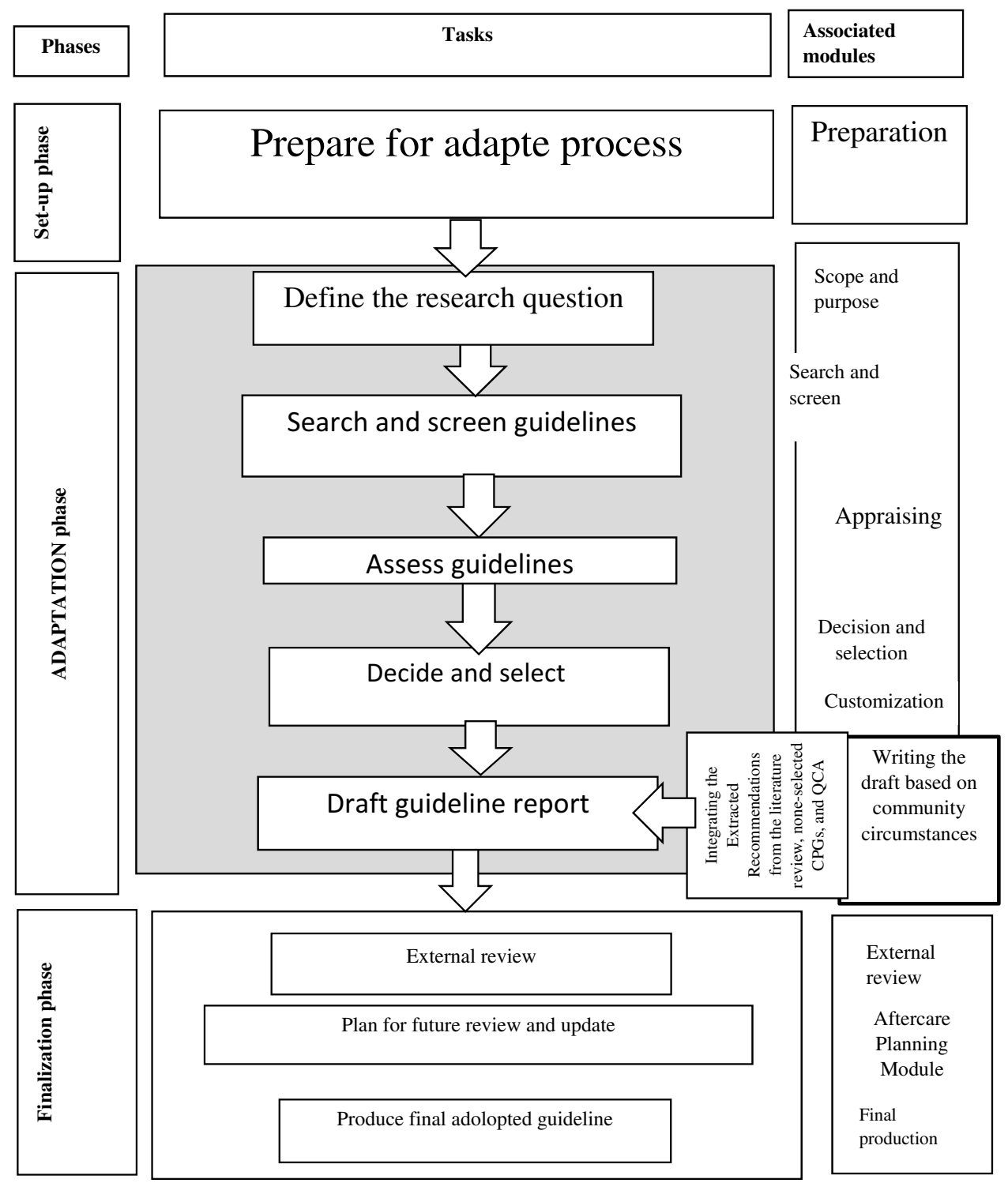

Figure I The adolopment process of this study.

establishing panel experts, development and progress of an adaptation plan. ${ }^{13}$ In the present study, the organizing committee included two professors, an associate professor, and a Ph.D. student. These individuals were involved in determining the scope of the investigation, assigning responsibilities, appointing members of the group of specialists and the expert panel, arranging meetings, and preparing a program for the adaptation process. These members participated in the specialist group, the expert panel, and the adaptation process.

\section{The Selection of the Guideline Theme}

Several criteria were considered in the selection of guideline theme, including the increasing prevalence of cancer in the Iranian society in both sexes, ${ }^{21}$ the disease burden on the health care system, family and community, and the prevalence of the CTIM (chemotherapy-induced mucositis), unsystematic variations in nursing interventions, and out-ofdate routine cancer nursing interventions in some nonstandard settings, and over-use, under-use or misuse of interventions in this regard, as witnessed by the research team members in Iranian clinical oncology, chemotherapy, and radiotherapy settings. Nursing practice variations lead to concerns about unavailability of baseline data on current nursing practices, the costs associated with different nursing practices, lack of standard and uniform procedures for the care of these patients; therefore, this guideline can be applicable in improving current nursing practice, it can prevent varied nursing performance, and improve care quality and/ or patient-related outcomes (e.g. quality of life or survival). ${ }^{9}$ Regarding the last item of this step, the availability of relevant 
high-quality evidence-based guidelines ${ }^{13}$ is a prerequisite for the adaptation process. Given there were no nursing nonpharmacological management CPGs, an innovative approach was used in the present study, in which non-pharmacological interventions as mentioned in eligibility criteria were extracted from relevant medical guidelines.

\section{The Identification of the Necessary Facilities and Skills} After it was ensured that there are extant CPGs to support the adaptation process, sufficient resources and preparations were needed to complete this process which included the following:

The panel members' commitment to attend in the discussion session to review all relevant documents, the obtainment of the necessary funds for the attendance of the panel members, including travel and accommodation expenses, presents for panel members in appreciation of their time and efforts for appraising CPGs, and the project management and administrative support were available for meeting coordination, documentation, storage and guideline collection. In the present study, the final guideline was not experimentally evaluated and only adolopted. In addition, individuals from key stakeholders were selected to form the panel. Panel members were selected from the following groups: healthcare professionals with clinical knowledge and skills on the guideline topic, cancer patients with a history of cancer therapy-induced mucositis, family caregivers of such cancer patients, health administration and policymaking experts, methodological experts on guideline development and appraisal, and individuals with managerial and facilitation skills in cancer care, to help the panel function effectively. In the case of patients and caregivers, their views and preferences were taken into account. Unfortunately, the panel did not include implementation experts, because no such professionals could be found in the setting of the present study.

It should be noted that the use of opinions from various experts involved in the care and treatment of patients will increase the authoritativeness of the guideline in clinical use, the applicability of the evidence-based recommendations of the guideline, and the positive impact of the guideline on patients' quality of life.

The sponsoring organization in this research was Medical University of Isfahan (MUI), Isfahan, Iran. The study was conducted within the timeframe set by and the funds allocated to the project by MUI.

According to Fitch et al, the number of individuals who attend the expert panel should be at least 7 and at most 15 , so that everyone has the opportunity to participate in the discussions. $^{22}$

\section{The Completion of Tasks of the Set-Up Phase}

The following items were considered:

1. Terms of collaboration: The scope of the project, membership, time commitment by panel members, and the frequency of the meetings were determined.

2. Declaration of conflicts of interest: All panel members were asked about conflicts of interest and they disclosed that they did not have any real conflicts of interest.

3. Consensus process: The organizing committee determined how decisions would be managed and how the findings of this process would be integrated with findings obtained from other sources in the final designed guideline.

4. The authorship of guideline: The organizing committee decided that the first author would be responsible for writing the guideline's draft.

5. The potential endorsing bodies of the final guideline: The organizing committee determined that panel members would review and endorse the designed guideline. Additionally, this guideline would be sent to the standardization department of the Ministry of Health for endorsement.

6. Dissemination and implementation strategies: The organizing committee decided to publish some articles and a booklet based on the final report. Moreover, the final report was incorporated into a Ph.D. Dissertation. However, this guideline has not yet been implemented, although the recommendations seem to be clear, objective, and implementable.

\section{Writing the Adaptation/Design Plan}

In this step, the research team wrote the guideline adaptation/design plan in which the target headings and the adaptation process were determined, including introduction, the subject area, the name of panel members, conflict of interest declarations, credentials of panel members, panel terms of reference, completion timeline and meeting schedule, funding source (Vice Chancellorship for Research of MUI).

During this process, the conclusions reached by the organizing committee and panel members were transparently documented. The first author coordinated and conveyed the details of this plan to all members of the panel and the expert group and contacted panel members by emails, letters, and telephone calls.

\section{Adaptation Phase}

This stage includes five steps as follows: 


\section{Determining the Health Questions}

The organizing committee applied the PIPOH components (i.e. patient population, intervention(s) of interest, professionals/patients, outcomes, healthcare setting, and context) to define the health question.

The patient population consisted of Iranian adult cancer patients undergoing chemotherapy. The interventions of interest were non-pharmacological care for cancer therapy-induced mucositis. The professional target population were nurses who work in chemotherapy wards, homecare nurses, family physicians, and oncologists.

The expected outcomes included improved quality of nursing care, increased patient satisfaction, improved costeffectiveness of care or treatment, reduction of hospital readmissions, and better coordination among treatment team members. This guideline is implementable in chemotherapy, oncology, and radiotherapy wards. In this study, the main research question was

What types of non-pharmacological interventions should be performed for cancer therapy-induced mucositis in an adult patient population undergoing chemo/radiotherapy?

\section{Searching Guidelines and Other Relevant Evidence}

A systematic search was conducted by researchers to find all relevant documents and guidelines published before Oct 2018 to be presented to panel members. For this purpose, the researchers conducted a systematic search on valid guideline websites from various countries, such as NICE, NGC, SIGN, NHSC, NGC, etc. and valid international databases, such as ScienceDirect, PubMed, ProQuest, CINAHL, Cochrane Library, Scopus, and Iranian databases, such as SID, Magiran, Irandoc, etc. to find eligible guidelines by using keywords which have been mentioned in Table 1.

\section{Screening of Retrieved CPGs}

After the search, several studies were found related to the subject and the main question. Due to time constraints and to reduce the workload, the retrieved documents were screened to select the best guidelines. To this end, first, two members of the research team (SS and FT) reviewed and screened the retrieved CPGs based on the eligibility criteria (diagram1). Then, these screened guidelines were appraised using the AGREE II (Appraisal of Guidelines for Research \& Evaluation II (AGREE II)) instrument and CPGs obtaining the highest quality scores based on this instrument were selected for the adaptation process.

\section{Eligibility Criteria}

It was decided to include structured CPGs addressing chemotherapy-induced mucositis among a target patient population of adults ( $\geq 18$ years old) published in the English language and providing non-pharmacological recommendations including prevention and management of mucositis in the study. All of the included CPGs were evidence-based and were original reports based on systematic reviews of related research evidence. We determined whether CPGs were evidence-based CPGs based on the search strategy reported in the guidelines and grading strength of evidence.

Expert opinion articles, literature reviews, systematic reviews, narrative reviews, protocols, clinical pathways, physician guide booklets, patients' guides, and editorials were excluded from the study.

It should be mentioned that occasionally an old guideline contains high-quality content and could be used to design a new guideline because finding new information in some fields might be difficult. In addition to the recommendations of the ADAPTE manual, in this study, the findings emerging from the literature review were used to fill in the gaps found in the selected guidelines.

It was attempted to offer clear and feasible recommendations as much as possible. However, some recommendations

Table I The Search Strategy Applied in This Study

\begin{tabular}{|c|c|c|c|c|c|c|}
\hline $\begin{array}{l}\text { Guideline } \\
\text { OR } \\
\text { Recommendation } \\
\text { OR } \\
\text { Standard } \\
\text { OR } \\
\text { Protocol } \\
\text { OR } \\
\text { Manuals } \\
\text { OR } \\
\text { Pathway }\end{array}$ & 更 & $\begin{array}{l}\text { Chemotherapy } \\
\text { OR } \\
\text { Radiotherapy }\end{array}$ & 更 & $\begin{array}{l}\text { Mucositis } \\
\text { OR } \\
\text { Oral complication } \\
\text { OR } \\
\text { Oral care } \\
\text { OR } \\
\text { Oral and dental health }\end{array}$ & AND & $\begin{array}{l}\text { Care } \\
\text { OR } \\
\text { Management } \\
\text { OR } \\
\text { Procedure }\end{array}$ \\
\hline
\end{tabular}


adopted from the selected guidelines obtained low scores from the appraisers in the CPG evaluation phase of the study, which could be explained by lack of the needed infrastructures in the Iranian healthcare system in comparison with the institutes in which guidelines originated.

\section{Revising Evaluations and Reducing the Number of Retrieved Guidelines}

Like related studies, the research team decided to select three CPGs for appraising. The three screened CPGs were evaluated by the AGREE instrument (see ref.,23). ${ }^{23}$

\section{The Assessment of Guideline Quality}

The quality of the selected CPGs were appraised using the AGREE instrument in order to select the base guidelines for consideration in the adaptation process.

\section{Preparing the Draft of the Adolopted Guideline (Figure I)}

Up to this stage, the ADAPTE protocol was followed, such that the needed recommendations were taken from the selected base CPGs. However, in this phase, a qualitative exploratory descriptive study was conducted to integrate the patients' care needs based on the views of the patients themselves, their families and caregivers, clinical nurses, oncologists, and radiotherapists. Furthermore, the selected guidelines were not the only sources that were used. That is, recommendations were also drawn from different valid sources and qualitative research studies, including randomized clinical trials (RCTs), review articles, non-selected guidelines, etc., to develop a comprehensive guideline. (Figure 2).

\section{Phase Three: Finalization}

In this phase, feedback was obtained on the guideline draft from target users of CPG. This was done in two steps:

First, a draft of the CPG was drawn up, the recommendations of which were selected from three sources, namely the screened and selected medical/nursing CPGs, literature review, and qualitative interviews with patients, their families, and nurses and physicians working in oncology wards. As one of the objectives of the study was to design a more comprehensive CPG than the basic CPGs found in the adaptation phase, this method was developed as an innovative approach to produce a nursing clinical practice guideline, which is applicable in cases where guidelines cannot be developed de novo or no related nursing guidelines are available for adaptation. Some nursing non-pharmacological recommendations were extracted from medical CPGs.
Second, we used a modified Delphi approach named the RAND/UCLA appropriateness method (RAM) ${ }^{22}$ consist of one survey and an expert panel as follows:

The drafted recommendations of the guideline were sent to a group of experts in questionnaire format (Table 2) to be appraised by experts in terms of relatedness, comprehensibility, usefulness, and feasibility.

In round one, which was conducted by mail in May 2017, expert group members were asked to evaluate each item on its suitability based on their knowledge and experience. Expert participants were asked to score the relatedness, comprehensibility, usefulness, and feasibility of each recommendation on a score range of 1-9. Then these scores were analyzed by SPSS 16 software and then by using descriptive statistics, the mean scores of expert panel's scores were accounted in mentioned four criteria, besides, the total mean score for each recommendation was accounted.

For data analysis, in this stage, the consensus of experts was applied to determine the suitability level of the recommendations based on the RAND/UCLA appropriateness method (RAM) as follows:

The recommendations were assigned to the "appropriate", "uncertain", and "inappropriate" nominal groups based on the obtained scores. A mean score of 1-3 was defined as inappropriate, 4-6 was defined as uncertain, and 7-9 was defined as appropriate. ${ }^{22}$

At the end of this stage, the recommendations which were identified as "appropriate", i.e., got a desirable score, were accepted and added to the CPG to prevent overloading the expert panel. Moreover, recommendations identified as "inappropriate" were omitted. Only recommendations that the expert group had not reached a consensus on were discussed in the expert panel.

In round two, 10 panelists met for a 1-day face-to-face meeting in June 2018 under the charge of two moderators (the first and the corresponding authors).

Panelists discussed each recommendation as a group and then they took one of four approaches: 1 . Rejection of the recommendation, 2. Adoption of the recommendation without modification, 3. Adaptation of the recommendation with modification, 4. Development of a new recommendation, and added the resulting recommendations to the CPG by consensus. Thus, the final adolopted clinical practice guideline was created by first author and the level of evidence/recommendations listed in this clinical practice guideline were determined according to Table 3 .

\section{Discussion}

To achieve a useful and holistic/comprehensive nursing CPG, adoption, adaptation, development processes were integrated with expert consensus, QCA methods, and 


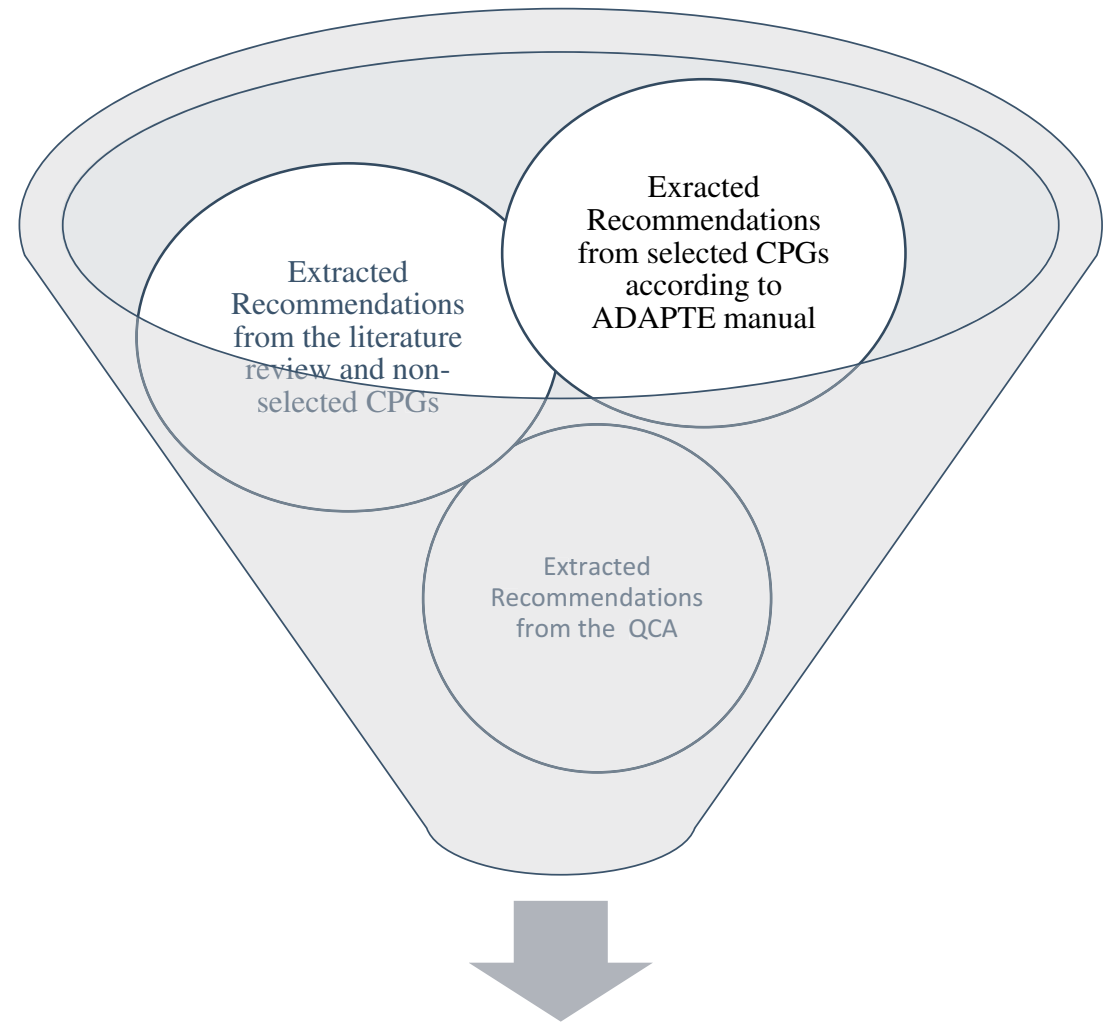

\section{The first draft of the CPG}

Figure 2 Integrating the recommendations extracted from the literature review, selected CPGs, none-selected CPGs, and QCA.

extensive literature search. Raine et al have also proposed a new approach for developing CPGs, because according to them CPGs are rarely based merely on research evidence, merging the expert consensus views in most cases ${ }^{24}$

In this study, a mix of three methods, i.e. the adoption, adaptation, and development of recommendations, was applied. A qualitative study and a systematic search were integrated within the adaptation approach of the recommendations, eventually, resulting in the development of an adolopted CPG for nurses. Schünemann et al, established an approach which they call "GRADE-ADOLOPMENT" of guideline recommendations that combines the advantages of adaptation, adoption and de novo guideline development. ${ }^{20}$ But they did not apply a QCA.
Given the paucity of nursing knowledge/NCPGs in this area, QCA was applied to generate rich recommendations with special regard to the Iranian context to address patient preference. Furthermore, the authors of the present study believe using a qualitative approach helps researchers obtain information about patients' preferences. Wallin said qualitative studies can provide highly valuable insights into nursing issues, yet such findings are often tricky to transform to guideline recommendations because of difficulties with generalization. ${ }^{11}$

A postal questionnaire was applied in the first round and a panel meeting was launched in the second round. Brook has used a postal questionnaire for the first round of ratings and a meeting for the second round, describing it as a hybrid approach. ${ }^{25}$

Table 2 The Nursing Interventions Questionnaire of Cancer Therapy Induced Mucositis

\begin{tabular}{|c|c|c|c|c|c|}
\hline Number & Recommendation & Relatedness (I-9) & Comprehensibility (I-9) & Usefulness (I-9) & Feasibility (I-9) \\
\hline \\
\hline \multicolumn{6}{|c|}{ - Column I: The row number, } \\
\hline \multicolumn{6}{|c|}{ - Column 2: The target intervention or recommendation } \\
\hline \multicolumn{6}{|c|}{ - Column 3: Relatedness, i.e. to what extent the proposed intervention is within the scope of nursing care } \\
\hline \multicolumn{6}{|c|}{ - Column 4: Comprehensibility, i.e. how easily can the written recommendation be understood } \\
\hline \multicolumn{6}{|c|}{ - Column 5: Usefulness which refers to how beneficial the proposed action is in achieving the established objective } \\
\hline \multicolumn{6}{|c|}{ - Column 6: Feasibility which means whether it is possible to implement such a recommendation. } \\
\hline \multicolumn{6}{|c|}{$\begin{array}{l}\text { Notable points: The group of experts was asked to separately rate the relatedness, comprehensibility, usefulness, and feasibility of each item. They were also asked to } \\
\text { suggest alternative wordings for the items if necessary. }\end{array}$} \\
\hline
\end{tabular}


Table 3 The Level of Evidence/Recommendations Listed in This Clinical Guideline

\begin{tabular}{|c|c|}
\hline $\begin{array}{l}\text { Level of } \\
\text { Evidence }\end{array}$ & Source of Evidence \\
\hline \multirow[t]{2}{*}{$A$} & $\begin{array}{l}\text { Recommendations extracted from clinical guidelines } \\
\text { published by accredited centers or from meta-analyzes } \\
\text { performed on randomized clinical trials }\end{array}$ \\
\hline & $\begin{array}{l}\text { Recommendations extracted from clinical guidelines } \\
\text { published by centers that have been critically accredited } \\
\text { through validated checklists or systematic review } \\
\text { studies conducted on randomized clinical trials. }\end{array}$ \\
\hline \multirow[t]{2}{*}{$B$} & $\begin{array}{l}\text { I. Recommendations extracted from reference text or evi- } \\
\text { dence extracted from clinical guidelines published by } \\
\text { other centers that have not been scientifically criticized } \\
\text { 2. Recommendations extracted from Systematic review } \\
\text { studies on non-randomized controlled trial }\end{array}$ \\
\hline & $\begin{array}{l}\text { Recommendations extracted from a case-control, case- } \\
\text { control, and cohort study }\end{array}$ \\
\hline \multirow[t]{2}{*}{$C$} & $\begin{array}{l}\text { Recommendations extracted from other articles with } \\
\text { the exception of Case report and Case series articles. }\end{array}$ \\
\hline & $\begin{array}{l}\text { I. Recommendations extracted from articles written } \\
\text { according to the consensus of experts. } \\
\text { 2. Recommendations extracted from Non-systematic } \\
\text { review articles }\end{array}$ \\
\hline \multirow[t]{2}{*}{ D } & $\begin{array}{l}\text { Recommendations extracted from articles written } \\
\text { based on expert clinical experience or from qualitative } \\
\text { study and panel members' opinions }\end{array}$ \\
\hline & $\begin{array}{l}\text { Recommendations extracted from articles based on } \\
\text { case reports, case series and correlational studies. }\end{array}$ \\
\hline
\end{tabular}

\section{Conclusion}

Given the paucity of NCPGs in the nursing management of cancer therapy-induced mucositis, and the lack of resources and time, and the difficulties of gathering skillful experts, the findings that emerged from the guideline adoption, adaptation, and development processes were integrated with the results obtained from expert consensus and the QCA method to achieve a more comprehensive nursing practice guideline in a developing country.

\section{Key Messages}

1. Since the development of CPGs requires financial resources and experts' skills, CPGs can be adapted accordingly to native conditions and local environments to standardize actions and optimize resource utilization.

2. There is a paucity of NCPGs in the nursing management of cancer therapy-induced mucositis, and the lack of cost, time and ability to gather skillful experts.
3. We integrate the findings emerged from adopt, Adapt, de novo guideline development by expert consensus and QCA method for achieving a more comprehensive nursing practice guideline in a developing country.

\section{Ethical Considerations}

This study was confirmed by the Vice-Chancellor in Research Affairs and Ethics Committee (code: 395651), Isfahan university of medical sciences, Iran.

This study was carried out in accordance with the principles of the Declaration of Helsinki. All of ethical considerations were addressed including patient data confidentiality and the written informed consent was obtained from all of participants for using their data in the study. Moreover we got permission for using "the ADAPTE manual and resource toolkit" from GI-N in this study.

\section{Acknowledgment}

This study is a part of a $\mathrm{PhD}$ thesis, which was done under the fund support of the Vice- Chancellor in Research of Isfahan University of Medical sciences (395651).

\section{Disclosure}

The authors report no conflicts of interest in this work.

\section{References}

1. Lenza NFB, Buetto LS, Vieira F, de Oliveira MS, Teles A, Sonobe HM. Intestinal colostomy needs in oncological monitoring: integrative review. J Nurs. 2015;9(6):8715-8724.

2. Rosenfeld BA. MOSFET dosimetry on modern radiation oncology modalities. Radiat Prot Dosimetry. 2002;101(1-4):393-398. doi:10.1093/oxfordjournals.rpd.a006009

3. Yarbro CH, Wujcik D, Gobel BH. Cancer Nursing. Jones \& Bartlett Publishers; 2016.

4. Scottish Intercollegiate Guidelines Network(SIGN). Guideline Developer's Handbook; 2011.

5. Nezamzadeh M, Khademolhosseini SM, Mokhtari Nori J, Ebadi A. Design of guidelines evidence-based nursing care in patients with angina pectoris. J Crit Care Nurs. 2012;4(4):169-176.

6. Bekkers R, Schuyt T. And who is your neighbor? Explaining denominational differences in charitable giving and volunteering in the Netherlands. Rev Relig Res. 2008;74-96.

7. Harrison MB, Légaré F, Graham ID, Fervers B. Adapting clinical practice guidelines to local context and assessing barriers to their use. Can Med Assoc J. 2010;182(2):E78-E84. doi:10.1503/cmaj.081232

8. Broadfield L. Best Practice Guidelines for the Management of Oral Complications from Cancer Therapy: Quick Reference Version. Cancer Care Nova Scotia; 2006.

9. Grol R, Hutchinson A, Eccles M, Grimshaw J. Clinical guidelines, potential benefits, limitations, and harms of guidelines. BMJ. 1999;318:527-530. doi:10.1136/bmj.318.7182.527

10. Penney GC. Adopting and adapting clinical guidelines for local use. Obstet Gynaecol. 2007;9(1):48-52. doi:10.1576/toag.9.1.048.27296

11. Wallin L. Clinical practice guidelines in nursing: a straightforward route to evidence-based practice? AWHONN Lifelines. 2005;9 (3):248-251. doi:10.1177/1091592305279125 
12. Decker TW, Cline-elsen J, Gallagher M. Relaxation therapy as an adjunct in radiation oncology. J Clin Psychol. 1992;48(3):388-393. doi:10.1002/ 1097-4679(199205)48:3<388::AID-JCLP2270480318>3.0.CO;2-O

13. The ADAPTE Collaboration. The ADAPTE process: resource toolkit for guideline adaptation. Version 2.0. Guideline international network. 2009. Available from: http://www.g-i-n.net. Accessed February 25, 2020.

14. Dabbagh A, Mirmiran B, Erfani N, et al. The appropriate approach for adoption of clinical practice guidelines in the national health system, according to the experienced project in Iran National Health System. Hakim Health Syst Res J. 2010;13(1):49-57.

15. Fervers B, Burgers JS, Haugh MC, et al. Adaptation of clinical guidelines: literature review and proposition for a framework and procedure. Int J Qual Health Care. 2006;18(3):167-176. doi:10. 1093/intqhe/mzi108

16. Kredo T, Machingaidze S, Louw Q, Young T, Grimmer K. South African Guidelines Excellence (SAGE): what' s in a name? $S$ Afr Med J. 2016a;106(1):18-20. doi:10.7196/SAMJ.2016.v106i1.10286

17. Smith B, Hender K, Frith P, Crockett A, Cheok F, Spedding S. Systematic assessment of clinical practice guidelines for the management of chronic obstructive pulmonary disease. Respir Med. 2003;97 (1):37-45. doi:10.1053/rmed.2002.1417

18. Husain SG, Cataldo TE. Late stomal complications. Clin Colon Rectal Surg. 2008;21(1):31. doi:10.1055/s-2008-1055319
19. Shekelle PG, Woolf SH, Eccles M, Grimshaw J. Clinical guidelines: developing guidelines. BMJ. 1999;318(7183):593-596. doi:10.1136/ bmj.318.7183.593

20. Schünemann HJ, Wiercioch W, Brozek J, et al. GRADE Evidence to Decision (EtD) frameworks for adoption, adaptation, and de novo development of trustworthy recommendations: GRADE-ADOLOPMENT. J Clin Epidemiol. 2017;81:101-110. doi:10.1016/j.jclinepi.2016.09.009

21. Ministry of Health and Medical Education. Iran cancer report in 2016. 2017.

22. Fitch K, Bernstein SJ, Aguilar MD, Burnand B, LaCalle JR. The RAND/UCLA Appropriateness Method User's Manual. Rand Corp Santa Monica CA; 2001.

23. Salarvand S, Hemati S, Adibi P, Taleghani F. A review of the quality of extant clinical practice guidelines in cancer therapy-induced mucositis. Int J Cancer Manag. 2017;10(10). doi:10.5812/ijcm

24. Raine R, Sanderson C, Black N. Developing clinical guidelines: a challenge to current methods. BMJ. 2005;331(7517):631. doi:10.1136/bmj.331.7517.631

25. Brook RH. The RAND/UCLA Appropriateness Method. Santa Monica, CA: RAND Corporation; 1995. Available from: https://www.rand.org/ pubs/reprints/RP395.html. Accessed February 25, 2020.

\section{Video abstract}

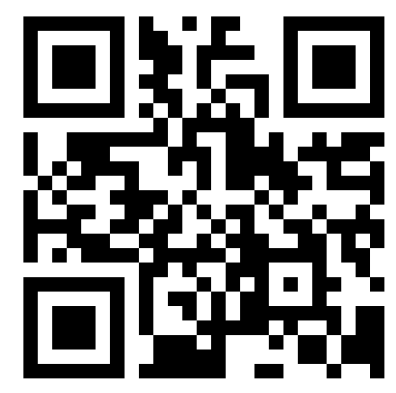

Point your SmartPhone at the code above. If you have a QR code reader the video abstract will appear. Or use: https://youtu.be/ADTV8ApyCXO
Cancer Management and Research

\section{Publish your work in this journal}

Cancer Management and Research is an international, peer-reviewed open access journal focusing on cancer research and the optimal use of preventative and integrated treatment interventions to achieve improved outcomes, enhanced survival and quality of life for the cancer patient.

\section{Dovepress}

The manuscript management system is completely online and includes a very quick and fair peer-review system, which is all easy to use. Visit http://www.dovepress.com/testimonials.php to read real quotes from published authors. 